\title{
Atomic Variational Calculations: Hydrogen to Boron
}

\author{
Frank Rioux \\ Department of Chemistry, St. John's University, College of St. Benedict, St. Joseph, MN 56374, \\ frioux@csbsju.edu
}

\begin{abstract}
Relatively simple quantum mechanical calculations are described for atoms and ions up to boron. The calculations employ scaled hydrogenic orbitals and can be completed with or without the aid of a computer. Given the simplicity of the trial wave functions the agreement with experiment is satisfactory.
\end{abstract}

Noting the absence of simple multielectron calculations in the pedagogical literature, Saleh-Jahromi and Moebs [1] recently described a one-parameter variational calculation on three-electron atomic species $\left(\mathrm{Li}, \mathrm{Be}^{+}, \mathrm{B}^{2+}\right)$ that gave reasonable agreement with experimental data. The purpose of the present manuscript is to demonstrate that it is easy to extend their calculation to four- and five-electron systems. Thus, without an unreasonable amount of mathematical effort it is possible to develop a set of one-parameter variational calculations on the elements from hydrogen to boron.

In this study one-, two-, and three-electron variational calculations will be reviewed and then extended to four- and five-electron systems. The scaled hydrogenic orbitals [2] that will be used are given below:

$$
\begin{gathered}
\Psi_{1 s}=\left(\frac{\alpha^{3}}{\pi}\right)^{1 / 2} \exp (-\alpha \mathrm{r}) \\
\Psi_{2 s}=\left(\frac{\alpha^{3}}{32 \pi}\right)^{1 / 2}(2-\alpha \mathrm{r}) \exp \left(-\frac{\alpha \mathrm{r}}{2}\right) \\
\Psi_{2 p_{z}}=\left(\frac{\alpha^{3}}{32 \pi}\right)^{1 / 2} \alpha \mathrm{r} \exp \left(-\frac{\alpha \mathrm{r}}{2}\right) \cos (\theta)
\end{gathered}
$$

Because the $1 \mathrm{~s}, 2 \mathrm{~s}$, and $2 \mathrm{p}_{\mathrm{z}}$ orbitals contain the same variational parameter $(\alpha)$ they remain orthogonal under the variational procedure. This prevents the need for an antisymmetric wave function and greatly reduces the mathematical complexity of the calculations. As we shall see later, this one-parameter simplicity comes at a price and the comparison between model and experiment deteriorates rapidly by the time we reach boron; however, there is still a lot of pedagogical merit in this set of calculations. Given the set of Mathcad-generated integrals in Appendix B ((42fr1897.zip Adobe Acrobat PDF or 42fr2897.zip Mathcad files), they require only the most rudimentary operations of differential calculus. Without the interference of complicated mathematics the students can see the basic quantum mechanical procedures more clearly.

The following is an outline of how the exercises might be carried out. a) Identify the contributions to the total electronic energy, $E$, of the species under study.

b) Go to Appendix B (42fr1897.zip Adobe Acrobat PDF or 42 fr2897.zip Mathcad files) to get the values of those contributions in terms of $\alpha$.

c) Minimize $E$ with respect to $\alpha$, and compare $E$ with the negative of the sum of ionization energies. See Table 1 .

d) Repeat steps 1 to 3 for the +1 ion and calculate the first ionization energy. Compare the calculated results with the experimental first ionization energies. See Table 1.

e) Calculate the orbital energies for all neutral species and compare the theoretical calculations with the orbital ionization energies. See Table 2.

How this set of tasks can be accomplished is outlined below.

\section{One Electron}

Two terms contribute to the total energy of a one-electron atom or ion: electron kinetic energy and electron-nucleus potential energy. Obviously Schrödinger's equation for the one-electron case can be solved exactly. However, for the sake of continuity with the multi-electron examples we note that if $\Psi_{1 \mathrm{~s}}$ is used as the trial function in a variational calculation the following expression for the energy is obtained.

$$
E=\left\langle T_{1 s}\right\rangle+\left\langle V_{N 1 s}\right\rangle=\frac{\alpha^{2}}{2}-Z \alpha
$$

Appendix A outlines the methods used to evaluate the electron-electron repulsion integrals required for multielectron atoms and ions. The evaluation of the $\left\langle T_{I s}\right\rangle$ and $\left\langle V_{N I s}\right\rangle$ integrals, as well all other integrals used subsequently, using Mathcad's symbolic processor is shown in Appendix B (42fr1897.zip Adobe Acrobat PDF or 42fr2897.zip Mathcad files). Minimization of $E$ with respect to $\alpha$ yields,

$$
\alpha=Z \text { and } E=\frac{Z^{2}}{2}
$$

For one-electron atoms and ions the total energy and the orbital energy of the electron are obviously the same. All calculations are in atomic units: $h=2 \pi ; m=e=4 \pi \varepsilon=1$. 
Table 1. Comparison of Atomic and Ionic Ground States with Experiment (3)

\begin{tabular}{llllll}
\hline & $\mathrm{H}$ & $\mathrm{He}$ & $\mathrm{Li}$ & $\mathrm{Be}$ & $\mathrm{B}$ \\
\hline $\mathrm{E}_{\text {atom }}$ & -0.500 & -2.848 & -7.235 & -14.062 & -23.317 \\
Exp. & -0.500 & -2.903 & -7.478 & -14.668 & -24.658 \\
$\%$ Error & 0 & 1.9 & 3.2 & 4.1 & 5.4 \\
E $_{\text {ion }}$ & -0.000 & -2.000 & -7.223 & -14.066 & -23.697 \\
Exp. & 0.000 & -2.000 & -7.283 & -14.332 & -24.363 \\
$\%$ Error & 0 & 0 & 0.82 & 1.9 & 2.7 \\
I.E. & 0.500 & 0.848 & 0.012 & -0.004 & -0.380 \\
Exp. & 0.500 & 0.904 & 0.198 & 0.343 & 0.305 \\
$\%$ Error & 0 & 6.2 & 94 & 100 & 230 \\
\hline
\end{tabular}

\section{Two Electrons}

There are five contributions to the total energy of a twoelectron atom or ion: the kinetic energy of each electron, the interaction of each electron with the nucleus, and the interaction of the electrons with each other. Using $\Psi_{1 \mathrm{~s}}$ as a trial function yields the following expression for the total energy.

$$
E=2\left\langle T_{1 s}\right\rangle+2\left\langle V_{N 1 s}\right\rangle+\left\langle V_{1 s 1 s}\right\rangle=\alpha^{2}-2 Z \alpha+\frac{5}{8} \alpha
$$

Minimization of $E$ with respect to $\alpha$ gives

$$
\alpha=Z-\frac{5}{16} \text { and } E=-\left(Z-\frac{5}{16}\right)^{2}
$$

For a two-electron atom or ion the orbital energy of an individual electron is the sum of its kinetic energy, its interaction with the nucleus, and its interaction with the other electron.

$$
E=\frac{\alpha^{2}}{2}-Z \alpha+\frac{5}{8} \alpha=-\frac{Z^{2}}{2}+\frac{5}{8} Z-\frac{75}{512}
$$

\section{Three Electrons}

The electronic structure of $\mathrm{Li}$ or a three-electron ion is $1 \mathrm{~s}^{2} 2 \mathrm{~s}^{1}$. There are nine contributions to the total energy: the kinetic energy of each electron (3), the interaction of each electron with the nucleus (3), and the electron-electron interactions (3).

$$
E=2\left\langle T_{1 s}\right\rangle+2\left\langle V_{N 1 s}\right\rangle+\left\langle V_{1 s 1 s}\right\rangle+\left\langle T_{2 s}\right\rangle+\left\langle V_{N 2 s}\right\rangle+2\left\langle V_{1 s 2 s}\right\rangle
$$

Consultation of Appendix B (42fr1897.zip Adobe Acrobat PDF or 42fr2897.zip Mathcad files) yields the following variational expression for $E$ in terms of $\alpha$.

$$
E=1.125 \alpha^{2}-2.250 Z \alpha+1.045 \alpha
$$

Minimization of $E$ with respect to $\alpha$ yields the following values for $\alpha, E$, and the 1 s and 2 s orbital energies.

$$
\begin{aligned}
& \alpha=Z-0.464 \\
& E=-1.125(Z-0.464)^{2} \\
& \epsilon_{1 s}=-0.500 Z^{2}+0.835 Z-0.280 \\
& \epsilon_{2 s}=-0.125 Z^{2}+0.420 Z-0.168
\end{aligned}
$$

\section{Four Electrons}

The $\mathrm{Be}$ atom electronic structure is $1 \mathrm{~s}^{2} 2 \mathrm{~s}^{2}$. The ground state energy consists of the following contributions.

$$
\begin{aligned}
& E=2\left\langle T_{1 s}\right\rangle+2\left\langle V_{N 1 s}\right\rangle+\left\langle V_{1 s 1 s}\right\rangle+ \\
& 2\left\langle T_{2 s}\right\rangle+2\left\langle V_{N 2 s}\right\rangle+4\left\langle V_{1 s 2 s}\right\rangle+\left\langle V_{2 s 2 s}\right\rangle
\end{aligned}
$$

Using Appendix B ((42fr1897.zip Adobe Acrobat PDF or 42fr2897.zip Mathcad files) we find after collecting terms

$$
E=1.250 \alpha^{2}-2.500 Z \alpha+1.615 \alpha
$$

Minimization of $E$ with respect to $\alpha$ yields the following values for $\alpha, E$, and the 1 s and 2 s orbital energies.

$$
\begin{aligned}
& \alpha=Z-0.646 \\
& E=-1.250(Z-0.646)^{2} \\
& \epsilon_{1 s}=-0.500 Z^{2}+1.045 Z-0.466 \\
& \epsilon_{2 s}=-0.125 Z^{2}+0.570 Z-0.316
\end{aligned}
$$

\section{Five Electrons}

The electronic configuration for boron under the present model is $1 s^{2} 2 s^{2} 2 p^{1}$. The $2 p_{z}$ orbital has been chosen for mathematical convenience, but the $2 p_{x}$ and $2 p_{y}$ orbitals would give the same result. The contributions to the boron groundstate energy are

$$
\begin{aligned}
& E=2\left\langle T_{1 s}\right\rangle+2\left\langle V_{N 1 s}\right\rangle+\left\langle V_{1 s 1 s}\right\rangle+2\left\langle T_{2 s}\right\rangle+2\left\langle V_{N 2 s}\right\rangle+4\left\langle V_{1 s 2 s}\right\rangle+ \\
& \left\langle V_{2 s 2 s}\right\rangle+\left\langle T_{2 p}\right\rangle+\left\langle V_{N 2 p}\right\rangle+2\left\langle V_{1 s 2 p}\right\rangle+2\left\langle V_{2 s 2 p}\right\rangle
\end{aligned}
$$

$E$ as a function of $\alpha$ is

$$
E=1.375 \alpha^{2}-2.750 Z \alpha+2.425 \alpha
$$

Minimization of $E$ with respect to $\alpha$ yields the following values for $\alpha, E$, and the $1 \mathrm{~s}, 2 \mathrm{~s}$, and 2 p orbital energies

$$
\begin{aligned}
& \alpha=Z-0.882 \\
& E=-1.375(Z-0.882)^{2} \\
& \epsilon_{1 s}=-0.500 Z^{2}+1.288 Z-0.747 \\
& \epsilon_{2 s}=-0.125 Z^{2}+0.732 Z-0.549 \\
& \epsilon_{2 p}=-0.125 Z^{2}+0.810 Z-0.617
\end{aligned}
$$


Table 2. Comparison of Calculated Orbital Energies with Experiment (3)

\begin{tabular}{lllllc}
\hline & $\mathrm{H}$ & $\mathrm{He}$ & $\mathrm{Li}$ & $\mathrm{Be}$ & $\mathrm{B}$ \\
\hline $\mathrm{E}_{1 s}$ & -0.500 & -0.897 & -2.275 & -4.286 & -6.087 \\
Exp. & -0.500 & -0.904 & -2.384 & -4.380 & -7.351 \\
$\%$ Error & 0 & 0.7 & 4.5 & 2.1 & 7.4 \\
$\mathrm{E}_{2 s}$ & & & -0.033 & -0.036 & -0.014 \\
Exp. & & & -0.198 & -0.343 & -0.518 \\
$\%$ Error & & & 83 & 90 & 97 \\
E $2 p_{\text {Exp. }}$ & & & & 0.308 \\
$\%$ Error & & & & & 0.305 \\
\hline
\end{tabular}

Appendix C shows (42fr1897.zip Adobe Acrobat PDF or 42fr2897.zip Mathcad files) how the five-electron calculation can be executed using Mathcad.

\section{Discussion of Results}

The results of these calculations are compared with experimental data in Tables 1 and 2 [4]. Table 1 gives the theoretical values for the atomic ground states and the theoretical ground state of the +1 ion. The experimental atomic ground-state energy is the negative of the sum of the successive ionization energies. The experimental ground state of the ionic species is the negative of the sum of all of the successive ionization energies except the first ionization energy. These comparisons between theory and experiment are made in rows 3 and 6 of Table 1 . It is also possible to calculate a theoretical first ionization energy by subtracting the atomic ground-state energy from the ground-state energy of the ion. This value can be compared with the experimental ionization energy as is shown in row 9 of Table 1.

Note that while the ground state energies for the neutral atom and the +1 ion are in acceptable agreement with experimental data given the level of theory used, the calculation of the first ionization energy deteriorates seriously for $\mathrm{Li}, \mathrm{Be}$, and $\mathrm{B}$. This is due to the fact that the first ionization energy is, in these cases, a small difference between two rather large quantities. Thus, the modest errors in $E_{\text {atom }}$ and $E_{\text {ion }}$ are amplified in the calculation of the ionization energy, IE $=$ $\left(E_{\mathrm{ion}}-E_{\mathrm{atom}}\right)$. From the point of view of the theoretical model, the problem is that for $\mathrm{Li}, \mathrm{Be}$, and $\mathrm{B}$ we have inner-shell electrons and valence electrons; thus, a one-parameter calculation is simply not adequate. This is clearly seen for Be and $\mathrm{B}$ where the model predicts that the +1 ions are more stable than the neutral atoms. The vast majority of the energy is in the core electrons and so it would be desirable for each orbital to have its own variational parameter. This can be done, but it requires, as noted above, an antisymmetric wave function and the evaluation of overlap and exchange integrals, significantly increasing the complexity of the calculations. Appendix D (42fr1897.zip Adobe Acrobat PDF or 42fr2897.zip Mathcad files) shows how this two-parameter calculation (after evaluation of relevant integrals) can be done using Mathcad. For the one-parameter calculation on $\mathrm{Li}$ the optimum value of $\alpha$ was found to be 2.536 . In the twoparameter calculation the core electrons have $\alpha$ equal to 2.680 and the valence electron has $\beta$ equal to 1.868 ; the ground state energy is improved by $2.2 \%$. In other words the core electrons' orbital has shrunk closer to the nucleus and the valence electron's orbital has undergone an expansion.
Table 2 compares the calculated orbital energies with experimental orbital ionization energies [4]. This comparison is based on Koopmans = theorem [5], which states that the orbital ionization energy can be approximated by the absolute value of calculated orbital energy. This assumes that ionization occurs as a two-step process: frozen ionization followed by relaxation of the ion to its true ground state. Under favorable conditions the energy change accompanying relaxation is small. This comparison is telling, basically, the same story as Table 1. The one-parameter model does not do a good job on the valence electrons of $\mathrm{Li}, \mathrm{Be}$, and $\mathrm{B}$.

\section{Conclusion}

In the typical undergraduate course dealing with quantum chemistry, quantum mechanical exercises are restricted to oneand two-electron systems. The purpose of this paper has been to show that it is possible to extend the range of exercises to three, four, and five electrons at the atomic level without a great deal of additional computational effort.

\section{Appendix A}

The electron-electron potential energy is calculated by first evaluating the electric potential, $\Phi$, due to one of the electron distributions. This is not difficult for spherical charge distributions [6]. For example, at the radial distance, $r$, from the nucleus the electric potential due to a spherical 1s charge distribution is

$$
\Phi_{1 s}(r)=\frac{e}{r} \int_{0}^{r} \Psi_{1 s}^{2}\left(r^{\prime}\right) 4 \pi r^{\prime 2} d r^{\prime}+e \int_{r}^{\infty} \frac{\Psi_{1 s}^{2}\left(r^{\prime}\right)}{r^{\prime}} 4 \pi r^{\prime 2} d r^{\prime}
$$

where $e$ represents the electron charge. All of the charge within the spherical volume element of radius $r$ behaves as if it were located at the center of the sphere. This contribution is given by the first integral in $\Phi_{1 \mathrm{~s}}(r)$. The remaining charge beyond $r$ can be viewed as consisting of many hollow spherical charge shells of thickness $\mathrm{dr}^{\prime}$. The electric potential due to this charge is the same anywhere inside the hollow shells as it at the center of the sphere. This contribution is given by the second integral in $\Phi_{1 \mathrm{~s}}(r)$.

Given $\Phi_{1 \mathrm{~s}}(\mathrm{r})$ the electron-electron potential energy interaction between a $1 \mathrm{~s}$ and $2 \mathrm{~s}$ electron is obtained by evaluating the following integral.

$$
V_{1 s 2 s}=e \int_{0}^{\infty} \Phi_{1 s} \Psi_{2 s}^{2} 4 \pi r^{2} d r
$$

The electron-electron potential integrals presented in Appendix B (42fr1897.zip Adobe Acrobat PDF or 42fr2897.zip Mathcad files) were evaluated using this method.

\section{References and Notes}

1. Saleh-Jahromi, A.; Moebs, W. Eur. J. Phys. 1998, 19, 355.

2. Levine, I. N. Quantum Chemistry, 4th ed. Prentice Hall: Englewood Cliffs, 1991, pp 232-235. 
3. Theoretical and experimental quantities in Tables 1 and 2 are expressed in atomic units. The hartree, $E_{\mathrm{h}}$, is the atomic unit of energy. $\left(1 E_{\mathrm{h}}=27.211 \mathrm{eV}=2625.5 \mathrm{~kJ} \mathrm{~mol}^{-1}\right.$. $)$

4. Shirley, D. A.; Martin, R. L.; Kowalczyk, S. P.; McFeely, F. R.; Ley, L. Phys. Rev. B 1977, 15, 544.
5. Koopmanns, T. A. Physica 1993, 1, 104.

6. Pauling, L.; Wilson, E. B. Introduction to Quantum Mechanics; McGraw-Hill: New York, 1935, pp 446-447. 